\title{
FURAZOLIDONE AND SEROTONIN SYNDROME: IS THERE ANY ASSOCIATION?
}

doi: 10.1590/S1807-59322008000400024

Petros N Karamanakos

The so-called "serotonin syndrome" (SS) is a potentially serious condition of central and peripheral serotonergic hyperstimulation. It is caused by the administration of single drugs or combinations of drugs that elevate synaptic levels of serotonin. It can arise following both therapeutic doses and overdoses, while the most serious cases are usually caused by an unintended synergism between a selective serotonin reuptake inhibitor (SSRI) and one or several other medications possessing serotonergic activity, such as monoamine oxidase (MAO) inhibitors. ${ }^{1}$ However, sometimes these other drugs are not known to possess serotonergic activity, making diagnosis more difficult.

Furazolidone is one of the synthetic antimicrobial nitrofurans used in the treatment of diarrhoea or enteritis caused by bacteria. It may also be useful in treating traveller's diarrhoea, typhoid fever, cholera and salmonella infections. Moreover, due to its anti-H. pylori activity, ${ }^{2}$ it has been used in China for more than 20 years in the treatment of peptic ulcers. In countries such as Brazil that have a large population with low socioeconomic levels and high bacterial resistance to metronidazole, furazolidone is believed to be a valuable alternative for patients who need retreatment for $H$. pylori eradication. ${ }^{3}$

As early as the 1960s, furazolidone was shown to be an inhibitor of MAO activity. ${ }^{4}$ In support of these results, we recently demonstrated for the first time that furazolidone produces a tremendous increase in brain serotonin levels in Wistar rats. ${ }^{5}$ Thus, it seems that furazolidone possess serotonergic activity and its combination with other serotonergic agents could, at least in theory, result in SS. However, this is not widely known and there have been few follow-up studies, making it possible that SS associated with furazolidone could go unrecognised. Labelling for furazolidone typically warns about interactions with MAO inhibitors and "tranquilisers", but not SSRIs; in addition, no warnings are given regarding the possibility of SS with these potential interacting drugs.

In conclusion, I suggest that clinicians should be aware of the serotonergic properties of furazolidone, which should be prescribed very cautiously in patients under therapy with SSRIs, MAO inhibitors, or other medications that have a high probability of inducing SS. Due to the potentially serious nature of this condition, it seems prudent that physicians always monitor patients receiving combinations of serotonergic drugs and be alert to the possibility of "serotonergic duplication". Retrospective case studies should be undertaken to look for furazolidone-induced SS. Moreover, basic research efforts in animal models are needed to better clarify the interactions of furazolidone with other serotonergic agents. In the meantime, physicians, pharmacists and clinical pharmacologists should keep the possibility of SS in mind when faced with patients taking furazolidone and who present with characteristic findings; anyone with such cases should publish them in Medlinereferenced journals, ideally with free internet full-text access, so that the information can be disseminated world-wide. 


\section{REFERENCES}

1. Bijl D. The serotonin syndrome. Neth J Med. 2004;62:309-13.

2. Howden A, Boswell P, Tovey F. In vitro sensitivity of Campylobacter pyloridis to furazolidone. Lancet. 1986;2:1035.

3. Eisig JN, Silva FM, Rodriguez TN, Hashimoto CL, Barbuti RC. A furazolidone-based quadruple therapy for Helicobacter pylori retreatment in patients with peptic ulcer disease. Clinics. 2005;60: $485-8$
4. Palm D, Magnus U, Grobecker H, Jonsson J. Inhibition of monoamine oxidase by bacteriostatically active nitrofuran derivatives. Naunyn Schmiedebergs Arch Exp Pathol Pharmakol. 1967;256:281-300.

5. Karamanakos PN, Pappas P, Boumba VA, Thomas C, Malamas M, Vougiouklakis T, et al. Pharmaceutical agents known to produce disulfiram-like reaction: effects on hepatic ethanol metabolism and brain monoamines. Int J Toxicol. 2007;26:423-32. 